\title{
Design and rationale of ischaemia-driven complete revascularisation versus usual care in patients with non-ST- elevation myocardial infarction and multivessel coronary disease: the South Limburg Myocardial Infarction (SLIM) trial
}

\author{
T. F. S. Pustjens · B. Streukens · J. Vainer · B. Gho · A. W. Ruiters · M. Stein · M. Ilhan · L. Veenstra · R. Theunissen · \\ S. C. A. M. Bekkers · A. W. J. van't Hof $\cdot$ S. Rasoul
}

Published online: 17 September 2019

(C) The Author(s) 2019

\begin{abstract}
Aims To compare ischaemia-driven complete coronary revascularisation by percutaneous coronary intervention (PCI) with usual care in patients with nonST-elevation myocardial infarction (non-STEMI) and multivessel disease (MVD).

Methods The South Limburg Myocardial Infarction (SLIM) trial (NCT03562572) is an investigator-initiated, prospective, multicentre, randomised controlled trial that compares fractional flow reserve (FFR)guided complete revascularisation during the index procedure with usual care in non-STEMI patients with MVD. A total of 414 patients will be randomised in a 1:1 fashion. The primary endpoint is the composite of all-cause mortality, non-fatal myocardial infarction, and any revascularisation and stroke (MACCE) at 12 months. The secondary endpoints are: MACCE at 24 and 36 months, and the composite of cardiac death, myocardial infarction, any revascularisation, stroke, major bleeding and left ventricular ejection fraction below $45 \%$ at 12, 24 and 36 months. Furthermore, quality of life will be assessed by the Patient Health Questionnaire (PHQ-9) and the Short
\end{abstract}

T. F. S. Pustjens $(\bowtie) \cdot$ B. Gho · A. W. Ruiters $\cdot$ M. Stein

M. Ilhan · L. Veenstra · A. W. J. van't Hof · S. Rasoul Department of Cardiology, Zuyderland Medical Centre,

Heerlen, The Netherlands

t.pustjens@gmail.com

J. Vainer $\cdot$ M. Ilhan $\cdot$ L. Veenstra $\cdot$ R. Theunissen ·

A. W. J. van't Hof · S. Rasoul

Department of Cardiology, Maastricht University Medical Centre, Maastricht, The Netherlands

B. Streukens · S. C. A. M. Bekkers

Department of Cardiology and Radiology, Maastricht

University Medical Centre, Maastricht, The Netherlands
Form (36) Health Survey (SF-36) at 1 and 12 months of follow-up.

Conclusion The SLIM trial aims to provide evidence whether FFR-guided complete revascularisation by PCI is superior to usual care with respect to clinical outcomes (major adverse cardiovascular events) in non-STEMI patients with MVD.

Keywords Acute coronary syndrome $\cdot$ Non-STelevation myocardial infarction - Multivessel disease . Complete revascularisation

\section{Introduction}

Patients with ST-elevation myocardial infarction (STEMI) and multivessel disease (MVD) have a worse prognosis than STEMI patients with single-vessel disease [1]. Several randomised clinical trials and metaanalyses have shown that, compared with infarctrelated artery only PCI (IRA-PCI), multivessel percutaneous coronary intervention (MV-PCI) resulted in fewer major adverse cardiovascular events (MACE), mainly driven by a reduction in repeat revascularisation [2-5].

Compared with STEMI patients, patients with nonST-elevation myocardial infarction (non-STEMI) have a higher risk profile, a higher incidence of MVD and less favourable outcome [6, 7]. However, there is no clear evidence regarding the role of ischaemia-driven complete coronary revascularisation by PCI in patients with non-STEMI during the index procedure. Furthermore, both the American and the European guidelines are unclear as to which coronary revascularisation strategy is preferred in non-STEMI patients with MVD $[8,9]$. 
Previous studies showed that MV-PCI in nonSTEMI patients reduces follow-up revascularisation rates without affecting MACE [10-12]. However, others also found MACE reduction after MV-PCI [13, 14]. In the SMILE (Survival of Myocardial Infarction Long-term Evaluation) trial, MV-PCI during the index procedure in non-STEMI patients with MVD was superior to multistage PCI during the index hospitalisation in terms of major adverse cardiovascular and cerebrovascular events (MACCE). This was mainly due to repeat coronary revascularisation, without a significant effect on cardiac death and reinfarction [14]. In the above-mentioned trials, assessment of lesion severity was mostly performed visually and fractional flow reserve (FFR) measurements were only performed in approximately $25 \%$ of the patients.

Currently, FFR-guided revascularisation has become the standard for in-catheterisation laboratory assessment of flow-limiting lesions in patients with stable angina, but has also been shown to be reliable in patients with unstable angina and non-STEMI [15-17]. The beneficial effect of FFR-guided MV-PCI compared with standard angiography was proven in the large randomised, multicentre FAME (Fractional Flow Reserve Versus Angiography for Multivessel Evaluation) study $[18,19]$. In that study, it was demonstrated that all types of adverse events were decreased by $30 \%$ in the 1 st year after PCI when guided by FFR. The information provided by FFR is similar to that obtained with myocardial perfusion studies. However, it is more specific and masking of one ischaemic area by another, more severely ischaemic, zone is avoided since every artery or segment is analysed separately [20, 21]. In addition, FFR-guided revascularisation has been shown to be cost-effective [22]. A recent study has shown that FFR can reliably assess the haemodynamic severity of non-culprit coronary artery stenosis during the acute phase of STEMI [23]. Therefore, FFR may offer the same advantages in the decision-making process on revascularisation of the non-culprit artery in patients presenting with nonSTEMI, similarly as in stable coronary syndromes.

Taking the above into consideration, we expect that ischaemia-driven (FFR) complete percutaneous revascularisation of all significant stenoses in lesions of non-culprit arteries performed within the index PCI procedure will improve clinical outcomes compared to the usual care, guided by the discretion of the physician.

\section{Methods}

\section{Study design}

The South Limburg Myocardial Infarction (SLIM) trial is an investigator-initiated, multicentre, prospective, randomised clinical trial in non-STEMI patients with multivessel coronary artery disease amenable to treatment with PCI, in which 414 consecutive patients will be randomised in a 1:1 fashion, after completion of a successful culprit lesion PCI. In the IRA-PCI the identification of the culprit vessel will be performed according to electrocardiographic, echocardiographic and angiographic parameters as stated in the ESC guidelines [24]. The use of anatomic (intravascular ultrasound or optical coherence tomography) or functional (FFR) imaging modalities to assess the culprit lesion, or to rule out other mechanisms such as dissection or haematomas, will be left to the operator's discretion. A patient will be excluded if the culprit artery is uncertain. Radial access will be strongly rec-

Table 1 Inclusion and exclusion criteria

\begin{tabular}{|c|c|}
\hline Inclusion criteria & Exclusion criteria \\
\hline Age between 18 and 85 years & Left main coronary artery disease (stenosis $>50 \%$ ) \\
\hline Presenting with non-STEMI, to be treated with $\mathrm{PCl}$ of culprit lesion & Chronic total occlusion of a non-IRA \\
\hline $\begin{array}{l}\text { Presence of at least one stenosis of }>50 \% \text { in a non-IRA on QCA or } \\
\text { visual estimation of baseline angiography }\end{array}$ & $\begin{array}{l}\text { Complicated IRA treatment, e.g. extravasation, permanent no reflow after IRA treat- } \\
\text { ment (TIMI flow 0-1) and inability to implant a stent }\end{array}$ \\
\hline Non-IRA stenosis amenable for PCI treatment (operator's decision) & Indication for or previous coronary artery bypass grafting \\
\hline \multirow[t]{10}{*}{ Able and willing to give signed informed consent } & Known severe cardiac valve dysfunction requiring surgery or TAVI in follow-up period \\
\hline & Killip class III or IV during completion of culprit lesion treatment \\
\hline & Uncertain culprit lesion \\
\hline & Life expectancy of $<1$ year \\
\hline & Intolerance to aspirin, clopidogrel, prasugrel, ticagrelor or heparin \\
\hline & Gastrointestinal or genitourinary bleeding in previous 3 months \\
\hline & $\begin{array}{l}\text { Planned elective surgical procedure necessitating interruption of P2Y12 inhibitors } \\
\text { during first } 6 \text { months post-enrolment }\end{array}$ \\
\hline & Currently enrolled in another clinical trial \\
\hline & Pregnancy \\
\hline & Expected loss to follow-up \\
\hline
\end{tabular}


Fig. 1 Randomisation procedure. FFR fractional flow reserve, Non-IRA noninfarct-related artery, NonSTEMI non-ST-elevation myocardial infarction, $\mathrm{PCl}$ percutaneous coronary intervention

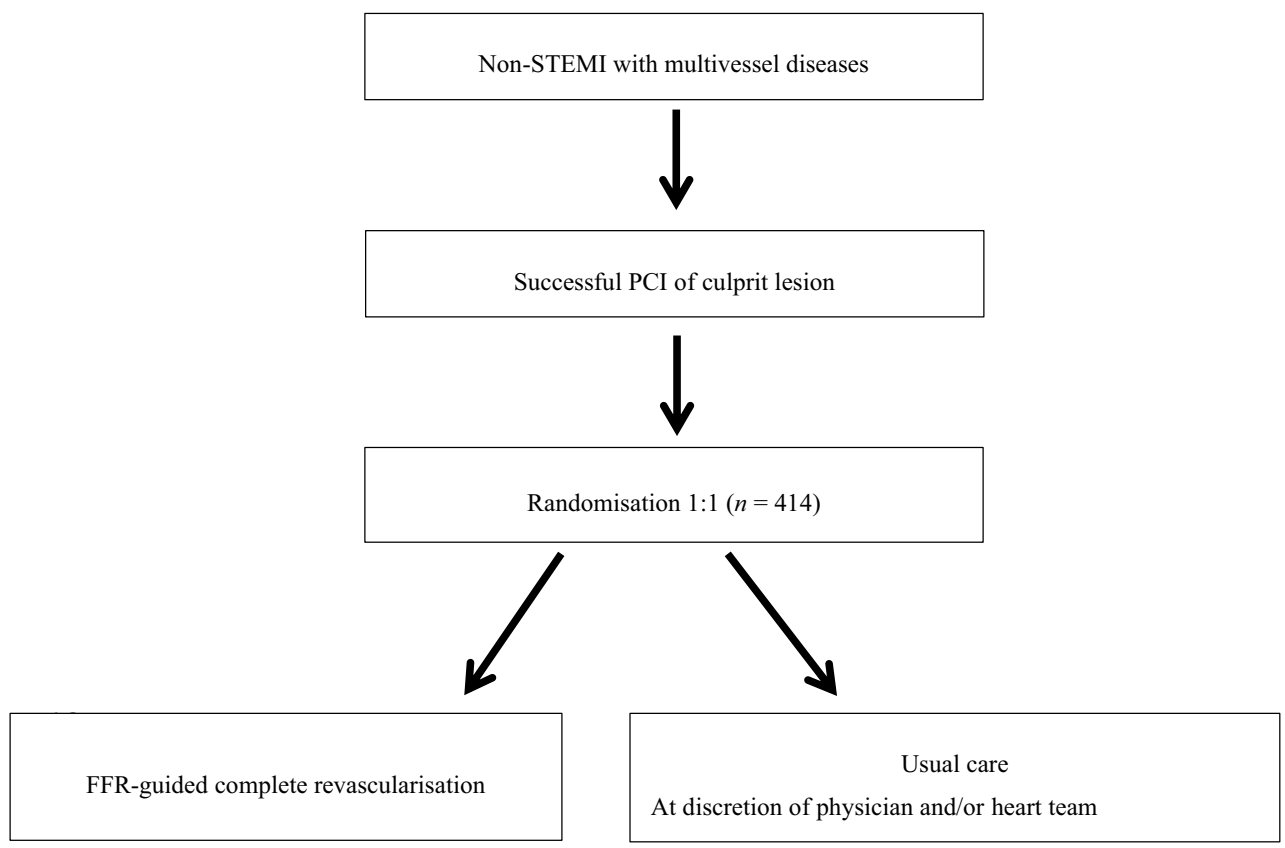

ommended for performance of coronary angiography and PCI.

All patients from June 2018 who present with at least one lesion with a stenosis of approximately $50 \%$ or more in a non-IRA with a diameter of $\geq 2.0 \mathrm{~mm}$ and fulfil the inclusion and exclusion criteria will be enrolled. Inclusion and exclusion criteria are listed in Tab. 1. The estimated duration of enrolment is 3 years. An overview of the trial is presented in Fig. 1.

The study has been approved by the Medical Ethical Committee of Zuyderland Medical Centre, The Netherlands. The SLIM trial is registered at ClinicalTrials.gov (NCT03562572) and the Netherlands Trial Register (NTR7158).

\section{Informed consent and randomisation}

Informed consent will be obtained according to the Good Clinical Practice guidelines. To participate in the trial, the participant must give oral approval before randomisation in the presence of a third independent (not involved in the study) person (catheterisation laboratory personnel or a nurse) after successful PCI of the culprit. To avoid a further delay in treatment, a signature will be obtained after full completion of the procedure irrespective of the treatment allocation, but must be obtained before the patient leaves the catheterisation laboratory. If angiographic inclusion criteria are met (presence of at least one stenosis of $>50 \%$ in a non-IRA with a diameter of $\geq 2.0 \mathrm{~mm}$ ) and PCI of the IRA is successful, the patient will be randomised via an online randomisation tool. If the patient is randomised to the usual care group, he or she will be informed that all intermediate lesions will be discussed in the heart team as to whether or not additional revascularisation of the non-IRA arteries will be performed. All patients will receive medical treatment based on current guidelines.

\section{Ischaemia-driven complete revascularisation group}

In the ischaemia-driven complete revascularisation strategy group all flow-limiting (FFR $\leq 0.80)$ lesions will receive treatment by PCI and stenting. The nonIRA PCI should be performed during the same intervention. Exceptions can be made for complex lesions where the operator estimates that the revascularisation procedure will require significant contrast overload, which may lead to deterioration of cardiac and renal function of the patient. In such cases, a second procedure should be performed, which should take place within the same hospitalisation, preferably within $72 \mathrm{~h}$.

\section{Usual care group}

In the randomised to usual care group the procedure will stop after the PCI of the culprit artery and the patient will be referred to his/her treating cardiologist and/or heart team. They will decide whether or not (ischaemia-driven) staged PCI of the non-IRA arteries should take place. The following treatment options are possible: (1) FFR- or iFR- (instantaneous wavefree ratio) guided PCI of the non-IRA arteries, (2) additional non-invasive tests, (3) symptom-driven PCI of the non-IRA arteries, or (4) optimal medical therapy. If the treating cardiologist (after consulting the heart team) decides to perform the non-IRA PCI revascularisation, then such treatment should take place within 6 weeks of the primary PCI in order to count as a scheduled staged PCI procedure. Any other revascularisations of any lesions after these 6 weeks are 
identified as unscheduled and therefore counted as an event.

All revascularisation procedures will be defined as clinically indicated or not clinically indicated by the independent clinical events committee.

\section{Study endpoints}

\section{Primary endpoint}

Primary study endpoints are defined as the incidence of MACE (composite endpoint of all cause death, nonfatal myocardial infarction, any revascularisation and stroke) at 12 months.

\section{Secondary endpoints}

- Primary endpoint in subgroups at 12 and 24 months.

- Composite endpoint of net adverse clinical events defined as composite endpoint of cardiac death, myocardial infarction, any revascularisation, stroke and major bleeding at 12, 24 and 36 months.

- Composite endpoint hospitalisation for heart failure and unstable angina pectoris at 12, 24 and 36 months.

- All-cause mortality or myocardial infarction at 12 , 24 and 36 months.

- Any revascularisation at 12,24 and 36 months.

- Stent thrombosis at 12,24 and 36 months.

- Bleeding (major and minor) at $48 \mathrm{~h}$ and 12 months.

- Primary endpoint at 36 months as well as outcomes of each component of the primary endpoint at 12 and 24 and 36 months.

- Left ventricular ejection fraction below $45 \%$ at 12,24 and 36 months (myocardial perfusion scintigraphy, magnetic resonance imaging or echocardiography).

- Quality of life at 1 and 12 months measured by the Patient Health Questionnaire (PHQ-9) and the Short Form (36) Health Survey (SF-36)

\section{Follow-up}

For endpoint adjudication office-based direct visits will be performed at 1 and 12 months, and telephone-based interviews will be performed at 24 and 36 months. At 1 and 12 months of follow-up, quality of life will be evaluated by the PHQ-9 and the SF-36.

\section{Statistical analysis}

\section{Sample size calculation and statistical power analysis}

The event rates for both groups were defined as follows:

In the usual care group, the event rates are estimated based on data from our previous all-comers registry [7] and the SMILE trial [13]. Rates of MACE in the ischaemia driven group are estimated based on the results of the SMILE trial [13].

In order to assess the superiority of MV-PCI over IRA-PCI, we assume the incidence of MACE to be $20 \%$ in the IRA-PCI group and $10 \%$ in the ischaemia-driven MV-PCI group. On the basis of a two-sided test size of $5 \%$ and a power of $80 \%$, it is calculated that a minimum of 197 patients is needed to be recruited in each group to detect a $10 \%$ difference in the incidence of MACE at 1 year. To account for $5 \%$ loss to follow-up, a total of $414(394 / 0.95)$ patients will be recruited.

The primary analysis is an intention-to-treat analysis of all randomised patients. A per-protocol analysis will also be performed. All continuous variables will be expressed as mean \pm SD and analysed by the Student's $t$-test. Categorical variables will be analysed by the chi-square or Fisher exact test, as appropriate. Baseline variables with non-Gaussian distributions will be compared using the Mann-Whitney $\mathrm{U}$ test and summarised with medians and interquartile range. For the primary endpoint and its components, 95\% confidence intervals will be reported. The event-free survival curve for MACE will be constructed using the Kaplan-Meier method, and statistical differences between curves will be assessed by the log-rank test. The hazard ratio for treatment comparisons will be estimated using Cox proportional hazard models. Mean scores of the health-related quality of life will be compared between groups using the independent $t$-test. Statistical analysis will be performed with SPSS (version 15.0, SPSS, Chicago, IL, USA). An independent endpoints committee blinded to the randomisation group will adjudicate clinical study endpoints.

Descriptive analyses of primary outcomes will be performed for the following pre-specified subgroups:

1. Diabetic patients versus non-diabetic patients

2 . Elderly ( $\geq 75$ years) versus young patients ( $<75$ years)

3. Male versus female gender

4. High- (GRACE >140) versus low-risk (GRACE $\leq 140$ ) patients according to Global Registry of Acute Coronary Events (GRACE) risk score

5. Patients with previous myocardial infarction versus patients with no previous myocardial infarction

\section{Interim analysis}

An interim analysis will be performed 6 months after 150 patients have been included. The interim analysis will be performed on the safety population and the actual treatment group will be unblinded to the independent statistician. All patients who are accrued and treated will be included in the analysis. Events of patients who are lost to follow-up, or who do not have 6 months of follow-up, will be included. The difference between groups in proportion of patients with events at 6 months of follow-up will be tested by means of a one-sided Fisher exact test for differences in independent binomial proportions. The data safety monitoring board will use the following stop- 
ping rule: If there is a significant difference in MACE between the two groups the trial will be stopped (onesided test, alpha $=0.05$ ).

\section{Trial coordination}

An independent Clinical Trial Centre will monitor the SLIM trial. The study monitor will visit each site at appropriate intervals to review investigational data for accuracy and completeness and ensure compliance with the protocol.

The study will be overseen by a trial steering committee and an independent Data and Safety Monitoring Board will review the clinical outcome data.

\section{Conclusion}

The SLIM trial is a prospective, multicentre, $1: 1$ randomised controlled trial in which 414 patients with non-STEMI and MVD will be included. The study aims to provide evidence whether FFR-guided complete revascularisation by PCI is superior with respect to clinical outcomes compared to usual care in nonSTEMI patients with MVD.

Funding The SLIM trial is an investigator-initiated study funded by an unrestricted grant from Abbott Vascular.

Conflict of interest A.W.J. van't Hof received research grants from Medtronic, Astra Zeneca, the Medicines Company, and Amgen and received speaker's fees from Astra Zeneca. T.F.S. Pustjens, B. Streukens, J. Vainer, B. Gho, A.W. Ruiters, M. Stein, M. Ilhan, L. Veenstra, R. Theunissen, S.C.A.M. Bekkers and S. Rasoul declare that they have no competing interests.

Open Access This article is distributed under the terms of the Creative Commons Attribution 4.0 International License (http://creativecommons.org/licenses/by/4.0/), which permits unrestricted use, distribution, and reproduction in any medium, provided you give appropriate credit to the original author(s) and the source, provide a link to the Creative Commons license, and indicate if changes were made.

\section{References}

1. Rasoul S, Ottervanger JP, de Boer MJ, et al. Predictors of 30day and 1-year mortality after primary percutaneous coronary intervention for ST-elevation myocardial infarction. Coron Artery Dis. 2009;20(6):415-21.

2. Wald DS, Morris JK, Wald NJ, et al. Randomized trial of preventive angioplasty in myocardial infarction. N Engl J Med. 2013;369(12):1115-23.

3. Kelly DJ, McCann GP, Blackman D, et al. Complete Versus culprit-Lesion only PRimary PCI Trial (CVLPRIT): a multicentre trial testing management strategies when multivessel disease is detected at the time of primary PCI: rationale and design. EuroIntervention. 2013;8(10):1190-8.

4. Engstrom T, Kelbaek H, Helqvist S, et al. Complete revascularisation versus treatment of the culprit lesion only in patients with ST-segment elevation myocardial infarction and multivessel disease (DANAMI-3-PRIMULTI): an open-label, randomised controlled trial. Lancet. 2015;386(9994):665-71.
5. Rasoul S, van Ommen V, Vainer J, et al. Multivessel revascularisation versus infarct-related artery only revascularisation during theindexprimaryPCIinSTEMI patients with multivessel disease: a meta-analysis. Neth Heart J. 2015;23(4):224-31.

6. Terkelsen CJ, Lassen JF, Norgaard BL, et al. Mortality rates in patients with ST-elevation vs. non-ST-elevation acute myocardial infarction: observations from an unselected cohort. Eur Heart J. 2005;26(1):18-26.

7. Rasoul S, Ottervanger JP, Dambrink JH, et al. Are patients with non-ST elevation myocardial infarction undertreated? BMCCardiovasc Disord. 2007;7:8.

8. Amsterdam EA, Wenger NK, Brindis RG, et al. 2014 AHA/ACC Guideline for the management of patients with non-ST-elevation acute coronary syndromes: a report of the American College of Cardiology/American Heart Association TaskForce on Practice Guidelines. JAm Coll Cardiol. 2014;64(24):e139-e228.

9. Authors/Task Force members, Windecker S, Kolh P, et al. 2014 ESC/EACTS Guidelines on myocardial revascularization: The TaskForce on Myocardial Revascularization of the European Society of Cardiology (ESC) and the European Association for Cardio-Thoracic Surgery (EACTS)Developed with the special contribution of the European Association of Percutaneous Cardiovascular Interventions (EAPCI). Eur Heart J. 2014;35(37):2541-619.

10. Brener SJ, Milford-Beland S, Roe MT, et al. Culprit-only or multivessel revascularization in patients with acute coronary syndromes: an American College of Cardiology National Cardiovascular Database Registry report. Am Heart J.2008;155(1):140-6.

11. Zapata GO, Lasave LI, Kozak F, et al. Culprit-only or multivessel percutaneous coronary stenting in patients withnon-ST-segmentelevation acutecoronarysyndromes: one-year follow-up. J Interv Cardiol. 2009;22(4):329-35.

12. Shishehbor MH, Lauer MS, Singh IM, et al. In unstable angina or non-ST-segment acute coronary syndrome, should patients with multivessel coronary artery disease undergo multivessel or culprit-only stenting? J Am Coll Cardiol. 2007;49(8):849-54.

13. Kim MC, Jeong MH, Ahn Y, et al. What is optimal revascularization strategy in patients with multivessel coronary artery disease in non-ST-elevation myocardial infarction? Multivessel or culprit-only revascularization. Int J Cardiol. 2011;153(2):148-53.

14. Sardella G, Lucisano L, Garbo R, et al. Single-staged compared with multi-staged PCI in multivessel NSTEMI patients: the SMILE trial. JAm Coll Cardiol. 2016;67(3):264-72.

15. Pijls NH, De Bruyne B, Peels K, et al. Measurement of fractional flow reserve to assess the functional severity of coronary-artery stenoses. NEngl J Med. 1996;334(26):1703-8.

16. Kern MJ, Lerman A, Bech JW, et al. Physiological assessment of coronary artery disease in the cardiac catheterization laboratory: a scientific statement from the American Heart Association Committee on Diagnostic and Interventional Cardiac Catheterization, Council on Clinical Cardiology. Circulation. 2006;114(12):1321-41.

17. Sels JW, Tonino PA, Siebert U, et al. Fractional flow reserve in unstable angina and non-ST-segment elevation myocardial infarction experience from the FAME (Fractional flow reserve versus Angiography for MultivesselEvaluation) study. JACC Cardiovasc Interv. 2011;4(11):1183-9.

18. Tonino PA, De Bruyne B, Pijls NH, et al. Fractional flow reserve versus angiography for guiding percutaneous coronary intervention. NEnglJ Med. 2009;360(3):213-24.

19. Pijls NH, Fearon WF, Tonino PA, et al. Fractional flow reserve versus angiography for guiding percutaneous coronary 
intervention in patients with multivessel coronary artery disease: 2-year follow-up of the FAME (Fractional Flow Reserve Versus Angiography for Multivessel Evaluation) study. JAm Coll Cardiol. 2010;56(3):177-84.

20. Lima RS, Watson DD, Goode AR, et al. Incremental value of combined perfusion and function over perfusion alone by gated SPECT myocardial perfusion imaging for detection of severe three-vessel coronary artery disease. J Am Coll Cardiol. 2003;42(1):64-70.

21. Pijls NH. Optimum guidance of complex PCI by coronary pressure measurement. Heart. 2004;90(9):1085-93.
22. Fearon WF, Nishi T, De Bruyne B, et al. clinical outcomes and cost-effectiveness of fractional flow reserve-guided percutaneous coronary intervention in patients with stable coronary artery disease: three-year follow-up of the FAME 2 trial (Fractional Flow Reserve Versus Angiography for MultivesselEvaluation). Circulation. 2018;137(5):480-7.

23. Smits PC, Abdel-Wahab M, Neumann FJ, et al. Fractional flow reserve-guided multivessel angioplasty in myocardial infarction. NEngl J Med. 2017;376(13):1234-44.

24. Neumann FJ, Sousa-Uva M, Ahlsson A, et al. 2018 ESC/ EACTS guidelines on myocardial revascularization. Eur Heart J.2019;40(2):87-165. 